\title{
Orthopaedic Sports Medicine Fellowship Websites: Information Considered Most Important to Fellowship Applicants
}

\author{
Christine M. McEvoy, B.A., Symone M. Brown, M.P.H., Cadence Miskimin, M.P.H., and \\ Mary K. Mulcahey, M.D.
}

\begin{abstract}
Introduction: The purpose of this study was to determine which components of sports medicine fellowships are most important to applicants when reviewing fellowship websites during the application process. Methods: An anonymous survey was distributed to 492 fellowship applicants from the 2017-2018 and 2018-2019 cycles. The survey included questions about the importance of including components of fellow education, recruitment, and experience on program websites. The weighted average of responses determined each component's rank, with 5 being "very important" and 1 being "not at all important." Responses were analyzed by application cycle, current position, and sex using the Wilcoxon rank-sum test. Results: Sixty-five applicants participated in the survey and completed the demographics section, resulting in a $13.2 \%$ response rate. According to participants, the most important components to include on fellowship websites were exposure to advanced operative sports medicine techniques (weighted average, 4.62), complexity of cases performed (4.52), and number of cases performed (4.50). Analysis demonstrated statistically significant differences in opinion between application cycles for flexibility for conducting a remote interview $(P=.0074)$, jobs obtained by previous fellows $(P=.019)$, national rank of department $(P=.021)$, program's geographic location $(P=.026)$, protected academic time $(P=.038)$, current positions for criteria for fellows' performance evaluations $(P=.028)$, program's geographic location $(P=.0097)$, and protected academic time $(P=.0079)$. There were statistically significant differences in opinion between current positions regarding flexibility for conducting a remote interview $(P=.0026)$, jobs obtained by previous fellows $(P=.012)$, and national rank of department $(P=.0013)$. Conclusions: Orthopaedic sports medicine fellowship applicants believe that it is most important to include information about the volume and complexity of fellows' cases and their day-to-day commitments on program websites. Clinical Relevance: This information would enable applicants to identify programs that will support professional development and allow program directors to communicate expectations.
\end{abstract}

$\boldsymbol{S}$ ubspecialization in orthopaedic surgery has increased significantly over the past 30 years. Recent studies suggest that more than $90 \%$ of graduating orthopaedic surgery residents complete at least 1 year of additional training and that $68 \%$ of open

From the Tulane University School of Medicine (C.M.E.) and Department of Orthopaedic Surgery (S.M.B., C.M., M.K.M.), Tulane University School of Medicine, New Orleans, Louisiana, U.S.A.

The authors report that they have no conflicts of interest in the authorship and publication of this article. Full ICMJE author disclosure forms are available for this article online, as supplementary material.

Received October 3, 2020; accepted January 24, 2021.

Address correspondence to Mary K. Mulcahey, M.D., 1430 Tulane Ave, \#8632, New Orleans, LA 70112, U.S.A. E-mail:mary.mulcahey.md@gmail. com

Published by Elsevier Inc. on behalf of the Arthroscopy Association of North America. This is an open access article under the CC BY-NC-ND license (http://creativecommons.org/licenses/by-nc-nd/4.0/).

2666-061X/201561

https://doi.org/10.1016/j.asmr.2021.01.015 positions require fellowship training. ${ }^{1,2,3}$ The San Francisco (SF) Match has facilitated the Orthopaedic Sports Medicine Fellowship Match annually since 2008. In 2019, there were 219 positions across 93 programs accredited by the American Orthopaedic Society for Sports Medicine and Arthroscopy Association of North America. ${ }^{4}$

Despite the competitive nature of the orthopaedic sports medicine fellowship application process, there is limited information available to applicants regarding fellowship programs. Applicants may be considering a wide range of program components when deciding which fellowship programs to apply, interview, and rank. Watson et al. ${ }^{3}$ proposed numerous reasons for the increase in fellowship training across surgical subspecialties, including additional training in advanced surgical technology, additional hours of more autonomous surgical experience, improved confidence and experience, and greater job marketability. Yayac 
et al. ${ }^{5}$ found that most fellowship program websites are difficult to access and provide insufficient information for applicants on the selection process. A previous study by Haislup et al. ${ }^{6}$ similarly noted that little information could be found on program websites regarding interview day structure, program expectations of fellows, and the selection committee's desired applicant qualities. The SF Match website only provides generalized guidelines regarding standards of professionalism during the interview cycle. ${ }^{4}$ Fellowship applicants could use more detailed and accessible information on program websites to rank programs based upon their career goals and personal interests more efficiently and effectively, leading to a more personalized and constructive training experience.

Out of the 9 recognized orthopaedic subspecialties, sports medicine has the highest number of applicants and is one of the most competitive. ${ }^{7}$ The purpose of this study was to determine which components of sports medicine fellowships are most important to applicants when reviewing fellowship websites during the application process.

We hypothesized that applicants would find details regarding the breath and volume of fellows' surgical experiences, the comparative rank of the fellowship program, and the program's research activities to be of greatest importance.

\section{Methods}

\section{Data Collection}

Before distributing the survey, approval was obtained from the institutional review board at our institution. An anonymous survey was distributed to all 492 applicants to the orthopaedic sports medicine fellowship who applied through the SF Match during the 2017-2018 and 2018-2019 cycles. The survey was distributed to the e-mail addresses applicants provided during the fellowship application process. The 13-question survey, which was hosted on SurveyMonkey (San Mateo, CA), took an average of 5 minutes to complete, gathered applicants' demographic information, and examined which components on a fellowship program website were considered most important to applicants.

The demographics section of the survey consisted of 9 individual questions regarding the participant's current academic position, sex, licensure as an allopathic or osteopathic physician, status as an international medical graduate (IMG), military affiliation, and age. This section also asked applicants about the geographic location of his or her residency program, his or her participation in 1 or both SF Match cycles, and the number of fellowship programs to which the participant applied during a cycle. The survey separated 39 potential website components into 3 categories: fellow education (13), fellow recruitment (16), and fellow experience (10). These components were identified after analyzing relevant literature for both orthopaedic subspecialty fellowship and other surgical subspecialties. ${ }^{2,3,5,8-13}$ Survey participants were asked to rank each component on a 5-point Likert scale, ranging from "not at all important" to "very important." The free response question at the end of the survey allowed participants to voluntarily share any other website information they considered important that was not included within one of the 3 categories.

\section{Data Analysis}

Descriptive statistics was used to analyze the data. The average of each website component response across all respondents was calculated and further analyzed by sex, application year, and current position. Each response on the Likert scale was given a weight ranging from 1 to 5: "not at all important" a rank of 1, "slightly important" a rank of 2, "moderately important" a rank of 3, "important" a rank of 4, and "very important" a rank of 5. These weights were used to calculate a weighted average, henceforth referred to as being "ranked," for each proposed website component. The data were analyzed to determine whether there was a difference in responses within 3 different demographic subgroups of applicants: (1) those who applied during the 2017-2018 cycle or 2018-2019 cycle, (2) those who were Post Graduate Year 5 (PGY-5) or current sports medicine fellow, and (3) those who were male or female. The Shapiro-Wilk test was used to determine whether the survey responses for each of these study population segments followed normal distributions. This was necessary because the distribution of data dictated which statistical test could be used to compare the similarity or differences of responses between each of the demographic subgroups. Because of the results of the Shapiro-Wilk test, the Wilcoxon rank-sum test was used to determine whether 2 sample populations differing by 1 demographic aspect wanted to see the same information on program websites. The null hypothesis for each Wilcoxon rank-sum test was that the median rank value for website components was the same between 2 sample subgroups. Analysis was completed using the packages ggplot2, survey, reshape2, and stringr in the programming language $R$ using the program RStudio (Boston, MA). The map was generated using Tableau Desktop version 2020.1 (Seattle, WA). The free response answers were reviewed and summarized.

\section{Results}

\section{Demographics}

A total of 65 applicants participated in the research study, which proceeded from October 14, 2019, to 
Table 1. Study Demographics $(n=65)$

\begin{tabular}{|c|c|}
\hline Characteristic & No. $(\%)$ \\
\hline \multicolumn{2}{|l|}{ Current position } \\
\hline PGY-4 & $0(0)$ \\
\hline PGY-5 & $30(46.2)$ \\
\hline Sports medicine fellow & $29(44.6)$ \\
\hline Other (please specify) & $6(9.2)$ \\
\hline \multicolumn{2}{|l|}{ Sex } \\
\hline Male & $49(75.4)$ \\
\hline Female & $16(24.6)$ \\
\hline Other (please specify) & $0(0)$ \\
\hline \multicolumn{2}{|l|}{ Medical degree program } \\
\hline Allopathic (M.D.) & $52(80.0)$ \\
\hline Osteopathic (D.O.) & $13(20.0)$ \\
\hline \multicolumn{2}{|l|}{ International medical graduate } \\
\hline Yes & $8(12.3)$ \\
\hline No & $57(87.7)$ \\
\hline \multicolumn{2}{|l|}{ Active duty military } \\
\hline Yes & $1(1.5)$ \\
\hline No & $64(98.5)$ \\
\hline \multicolumn{2}{|l|}{ Age, y } \\
\hline$<30$ & $2(3.1)$ \\
\hline $30-34$ & $54(83.1)$ \\
\hline $35-39$ & $6(9.2)$ \\
\hline $40-44$ & $3(4.6)$ \\
\hline $45+$ & $0(0)$ \\
\hline \multicolumn{2}{|l|}{ Match cycle } \\
\hline 2017-2018 & $26(40.0)$ \\
\hline 2018-2019 & $39(60.0)$ \\
\hline 2017-2018 and 2018-2019 & $0(0)$ \\
\hline \multicolumn{2}{|c|}{ Total number of fellowship applications } \\
\hline $1-5$ & $1(1.5)$ \\
\hline $6-10$ & $2(3.1)$ \\
\hline $11-15$ & $8(12.3)$ \\
\hline $16-20$ & $12(18.5)$ \\
\hline $21+$ & $42(64.6)$ \\
\hline
\end{tabular}

November 18, 2019, resulting in a response rate of $13.2 \%$. Thirty $(45.2 \%)$ participants held the position of PGY-5, and $29(44.6 \%)$ held the position of sports medicine fellow. Six $(9.2 \%)$ of the study participants selected other, clarifying that they are now attending physicians (2), are completing another fellowship before completing a sports medicine fellowship (2), or hold board certification in another country (2). Fortynine $(75.4 \%)$ participants were male, and $52(80.0 \%)$ participants had graduated from an allopathic medical school. All residency locations were represented by the respondents except the West-Mountain region. Nineteen $(29.2 \%)$ respondents attended residency in the Midwest-East North Central region, $11(16.9 \%)$ in the West-Pacific region, $9(13.9 \%)$ in the Northeast-Middle Atlantic region, $8(12.3 \%)$ in the Midwest-West North Central region, $5(7.7 \%)$ in the Northeast-New England region, 5 (7.7\%) in South-South Atlantic region, $5(7.7 \%)$ in the South-West South Central region, and $3(4.6 \%)$ in the South-East South Central region. Eight $(12.3 \%)$ of the respondents were IMGs, and 1 participant $(1.5 \%)$ reported serving as a physician in the US Armed Forces. Fifty-four $(83.1 \%)$ respondents were 30 to 34 years old. Twenty-six $(40 \%)$ of the participants applied during the 2017-2018 match cycle, while 39 (60\%) participants applied during the 2018-2019 match cycle. Forty-two $(64.6 \%)$ respondents reported submitting at least 21 applications when applying to the SF Match. These results can be found in Table 1 and Fig 1 .

\section{Fellowship Education}

Fifty-eight $(89.2 \%)$ survey participants completed a question asking them to rank certain components of fellow education that should be included on a program's website from most important to least important. Four of the components ranked above 4: exposure to advanced operative sports medicine techniques (4.62), complexity of cases performed (4.52), number of cases performed (4.5), and list of faculty members (4.4). The 3 components considered least important (score <3) were list of faculty members' published research and presentations (2.95), opportunity to teach residents (2.86), and journal club (2.52). These results can be found in Fig 2.

The Wilcoxon rank-sum test could not reject the null hypothesis that responses regarding components of fellow education did not vary between respondents of different application years, different positions, and different sexes. This is because the $P$ values for tests regarding all of these components were all greater than .05 .

\section{Fellowship Recruitment}

Fifty-five $(84.6 \%)$ survey participants completed the question asking participants to rank what information pertaining to recruitment should be included on a website from most important to least important. None of the components ranked above 4. Eleven of the components ranked between 3 and 4, with the 3 highest being list of current fellows (3.93), list of jobs obtained by previous fellows (3.89), and summary description of sports medicine fellowship program's values and expectations (3.89). The 3 lowest components ranked were link to SF Match website (2.11), link to American Orthopaedic Society for Sports Medicine website (2.09), and link to Arthroscopy Association of North America website (2.04). These results can be found in Fig 3.

The Wilcoxon rank-sum test rejected the null hypothesis, thus finding a difference of opinion between subgroups, for some components of fellow recruitment. There were statistically significant differences in opinion between application cycles for flexibility for conducting a remote interview $(P=.0074)$, list of jobs obtained by previous fellows $(P=.019)$, and national rank of orthopaedic surgery department $(P=.021)$. There were statistically significant differences in opinion between current positions for flexibility for 


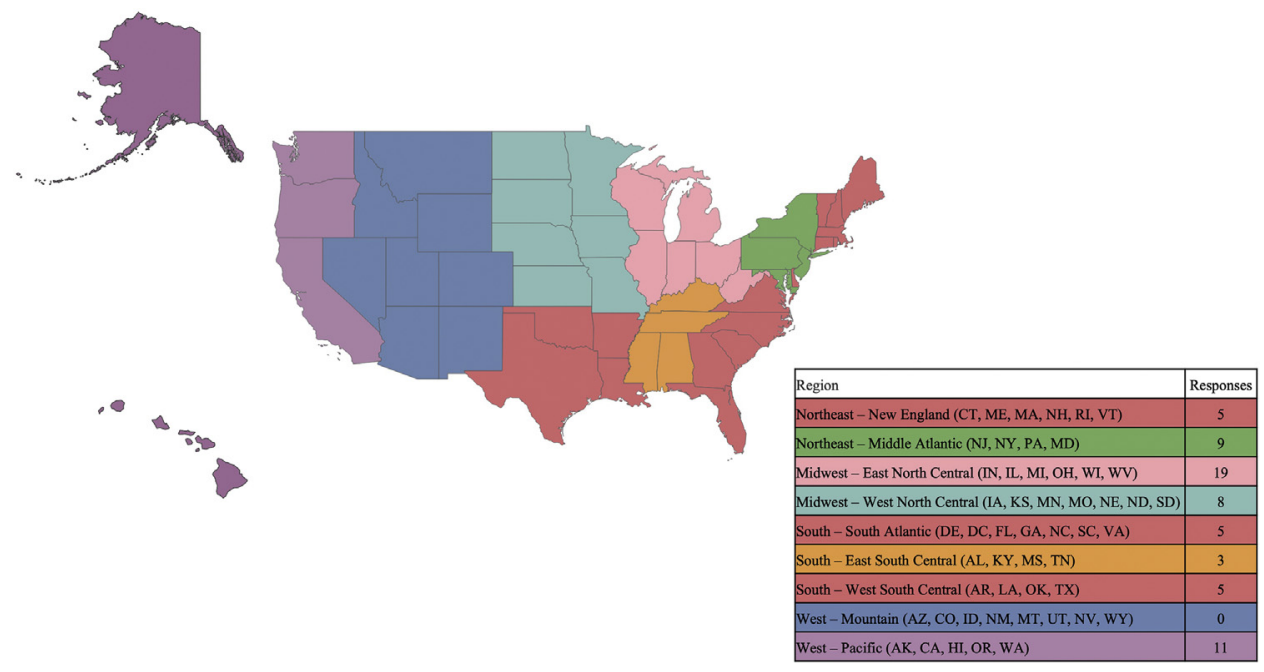

Fig 1. Survey respondents by geographic location. conducting a remote interview $(P=.0026)$, list of jobs obtained by previous fellows $(P=.012)$, and national rank of orthopaedic surgery department $(P=.0013)$. There were no identified differences in opinion between male and female applicants regarding all components of fellow recruitment.

\section{Fellowship Experience}

Fifty-four $(83.1 \%)$ survey participants responded to the question asking them to rank what information pertaining to fellow experience should be included on the website. Four of the components ranked above 4: team coverage (4.48), number of fellows per year
(4.44), rotation schedule (4.31), and information on external rotations $(4.06)$. The lowest-ranked component was criteria for fellows' performance evaluations (2.89). These results can be found in Fig 4 .

The Wilcoxon rank-sum test rejected the null hypothesis, thus finding a difference of opinion between subgroups, for some components of fellow experience. There were statistically significant differences in opinion between applicants in the 2 application cycles for program's geographic location $(P=.026)$ and protected academic time $(P=.038)$. There were statistically significant differences in opinion between participants of different current positions for criteria for fellows'

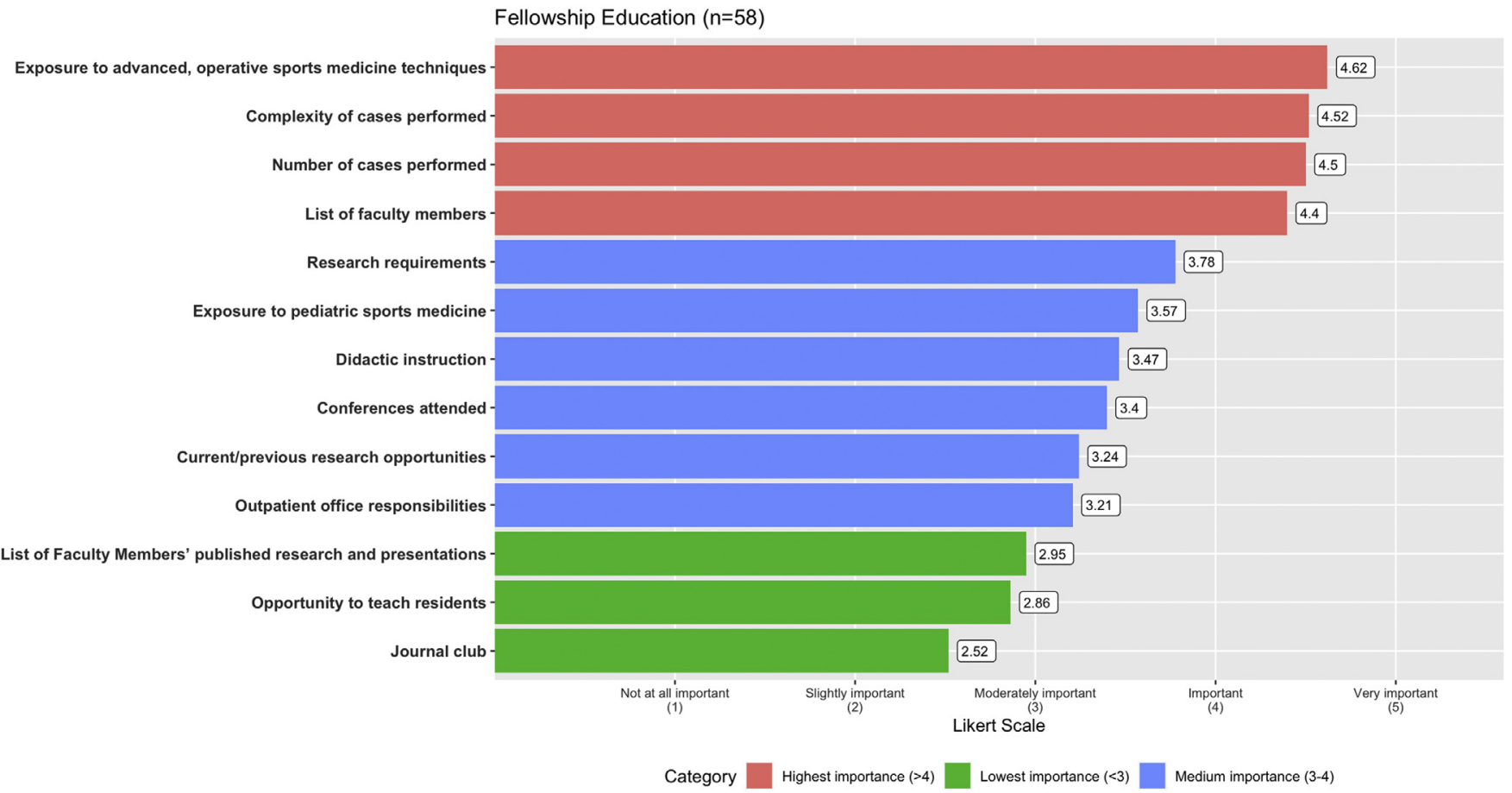

Fig 2. Weighted averages for responses regarding fellowship education. 


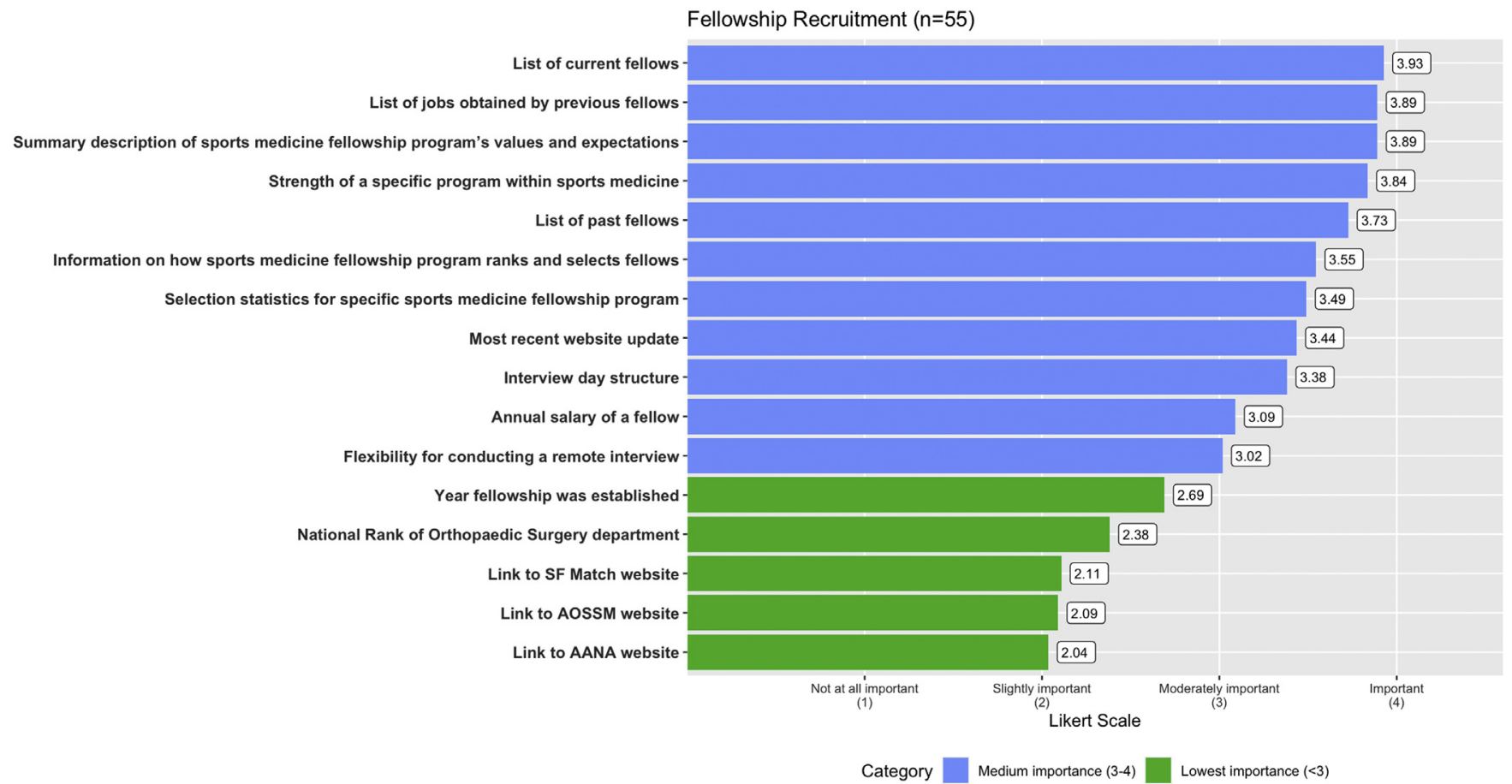

Fig 3. Weighted averages for responses regarding fellowship recruitment.

performance evaluations $(P=.028)$, program's geographic location $(P=.0097)$, and protected academic time $(P=.0079)$. There were no identified differences in opinion between male and female applicants regarding all components of fellow experience.

\section{Free Response}

Six $(9.23 \%)$ survey participants responded to the free response question that asked whether they felt there was anything else that should be included on a fellowship website that was not listed in the previous questions. One study participant $(1.5 \%)$ felt websites

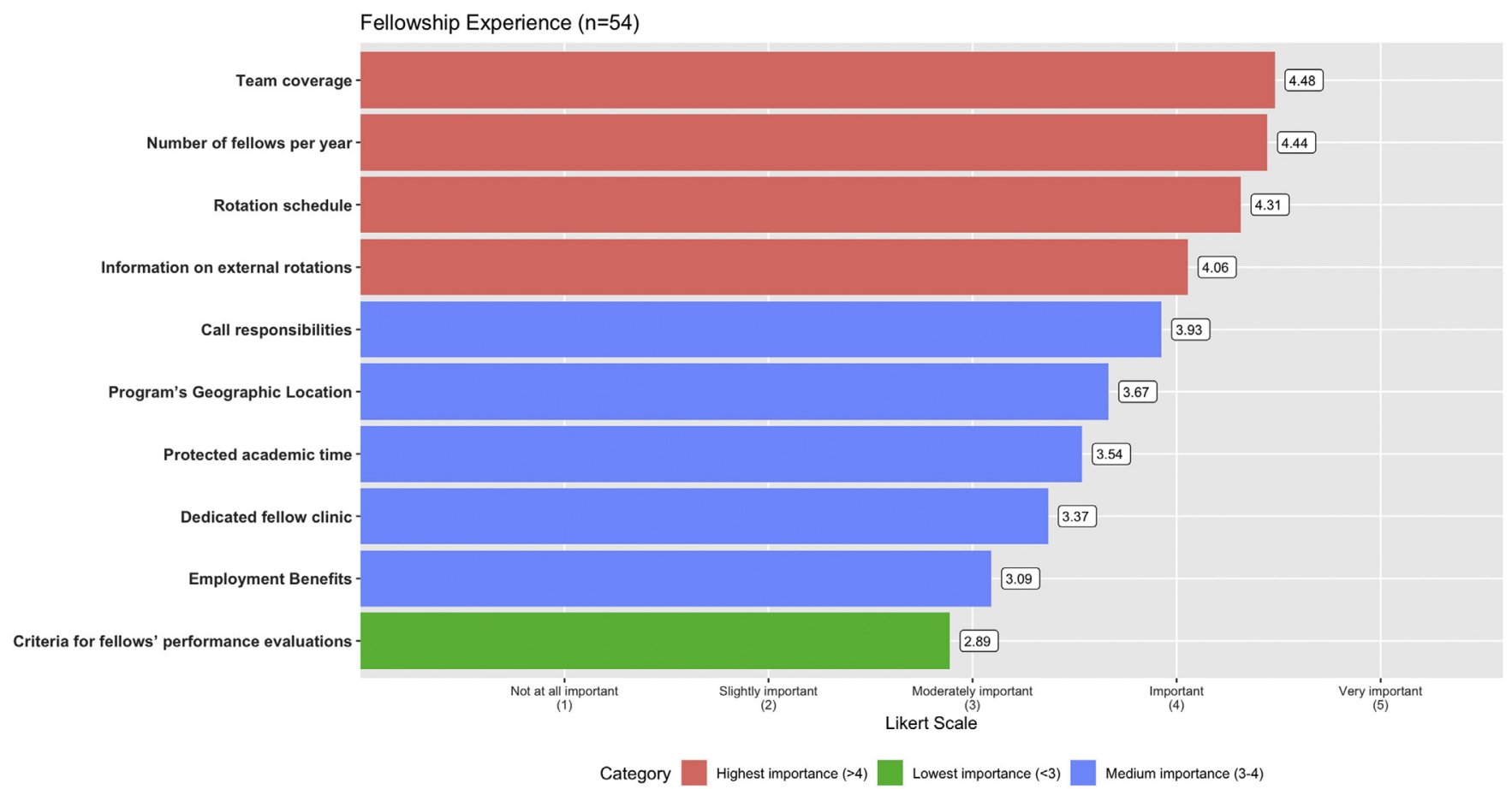

Fig 4. Weighted averages for responses regarding fellowship experience. 
should clearly describe team coverage expectations, while another participant wanted programs to make the application criteria for IMGs clearer on their websites. There was a comment specifically focused on "opportunities to do other cases outside the realm of sports medicine (joints, hand, F\&A)." Another participant wanted more specifics on the "ability to attend national conferences," specifically noting how many are allowed and/or required. Last, 1 participant noted his or her desire for the website to include interview dates and any extra application requirements outside the SF Match application.

\section{Discussion}

Applicants to sports medicine fellowship programs felt that 4 components of fellowship education (exposure to advanced operative sports medicine techniques, complexity of cases performed, number of cases performed, and list of faculty members) and 4 components of fellowship experience (team coverage, number of fellows per year, rotation schedule, and information on external rotations) were of highest importance to include on fellowship program websites.

\section{Fellowship Education}

Four components of fellowship education were of highest importance to study participants reviewing program websites, as these components had an average weight above 4: exposure to advanced operative sports medicine techniques, complexity of cases performed, number of cases performed, and list of faculty members. Shui et al. ${ }^{4}$ noted, "Orthopaedic education has faced recent challenges, such as duty-hour restrictions, increased documentation requirements, and an expanding technical skill set," and emphasized how time spent in the operating room is the most valued but significantly limited during orthopaedic residency. Similar studies examining the fellowship application process have surveyed applicants of neurotology, laryngology, and pediatric otolaryngology fellowships. All of these studies found that surgical exposure, volume of surgery, caseload, and refinement of endoscopic surgery skills were considered highest priority when evaluating fellowship programs. ${ }^{11-13}$ Applicant interest in program faculty is not unique to orthopaedic sports medicine fellowship applicants. Laryngology applicants considered a program's emphasis on mentorship a component of highest importance when evaluating programs, and applicants to pediatric otolaryngology fellowships considered otology faculty reputation a component of greatest importance. ${ }^{12,13}$ Programs could consider hyperlinking to a preexisting webpage on the academic institution's website to provide applicants with information about fellowship faculty. With regards to the fellowship education components of medium importance (average rank between 3 and 4), DeFroda et al. ${ }^{8}$ found that the average number of publications per applicant has increased every application cycle over the past 10 years. This study also found that applicants matching to programs in the Northeast region of the United States often had a higher number of publications (6.41 publications per fellow) than those matching to programs in other regions of the country. Cvetanovich et al. ${ }^{9}$ remarked that some applicants consider an orthopaedic sports medicine fellowship an opportunity to complete impactful research that will positively advance their careers, especially in future positions at academic medical centers. Research opportunities, requirements, and expectations could be stated on programs' websites with lists of host departments' publications and presentations. Although faculty members' published works received a weight of lower significance (2.95), including such information on a program's website could answer applicants' higher priority questions about research prospects.

\section{Fellowship Recruitment}

No component of fellow recruitment received an average weighted score above "important." Applicants may not consider recruitment as crucial for successful navigation of the fellowship application process because of the competitive nature of the orthopaedic sports medicine fellowship match. Applicants may have learned about a program's rank or strength through word of mouth or online forums, or they may focus on individual fit more than rank when evaluating fellowship programs. ${ }^{10}$ Programs could still include lists of both current and previous fellows, as well as jobs obtained by previous fellows, so they can be easily accessed by potential applicants. Sentiment differed between application years and applicant positions as either residents or fellows with respect to the ability to do a remote interview and the list of jobs obtained by past fellows. Opinions also differed between applicant positions in training regarding the rank of the orthopaedic surgery department. These observed differences between groups could be due to the sample size, as the unique circumstances of certain applicants one year or in one training position may have shifted the results. Sports medicine fellows may have felt differently after reflecting on the application process.

\section{Fellowship Experience}

Work-life balance has become an important topic of discussion throughout the medical profession. One survey study of 152 orthopaedic surgeons, who have held leadership positions (e.g., department chair or journal editor), found that $114(75 \%)$ respondents felt they had less time than they needed for their personal lives. ${ }^{14}$ Applicants may use a fellowship program's website description of the typical workweek to weigh how the program's culture may align with their own 
career goals and personal values. Opinions differed between application years for geographic location of the residency program and protected academic time. A difference was also observed between PGY-5 participants and current fellows regarding geographic location of the residency program, protected academic time, and specific information regarding performance evaluations. These observed differences between groups could be due to the sample size, as the unique circumstances of certain applicants one year or in one position may have shifted the results. Current sports medicine fellows also may have felt differently after beginning their respective fellowship programs.

\section{Limitations}

There are several limitations to this study. First was the method through which participants were recruited. Some prospective participants' e-mail addresses were from past institutions (i.e., their residency program) and may no longer be active or checked regularly. Second, past applicants may also have less incentive to participate in a study about a process they already completed. The relatively low response rate in this study may limit the generalizability of the results. The smaller pool of responses may have subsequently obscured potential statistical differences in opinion between, for example, male and female participants.

\section{Conclusions}

Orthopaedic sports medicine fellowship applicants believe that it is most important to include information about the volume and complexity of fellows' cases and their day-to-day commitments on program websites.

\section{References}

1. Mead M, Atkinson T, Srivastava A, Walter N. The return on investment of orthopaedic fellowship training: A tenyear update. J Am Acad Orthop Surg 2020;28:e524-e531.

2. Shiu B, Petkovic D, Levine W, Ahmad C. Maximizing surgical skills during fellowship training. J Am Acad Orthop Surg 2017;25:421-426.

3. Watson SL, Hollis RH, Oladeii L, Xu S, Poterfield JR, Ponce BA. The burden of the fellowship interview process on general surgery residents and programs. J Surg Educ 2017;74:167-172.

4. SF Match Residency and Fellowship Matching Service, https://www.sfmatch.org/SpecialtyInsideAll.aspx? $\mathrm{id}=3$ $2 \delta$ typ $=1$ \&name $=$ Sports $\% 20$ Medicine $\delta$ mainSpecialty $=0$ rthopaedics $\% 208$ subspec $=28$. Accessed October 23, 2019.

5. Yayac M, Javandal M, Mulcahey MK. Accredited orthopaedic sports medicine fellowship websites: An updated assessment of accessibility and content. Orthop $J$ Sports Med 2017;5:2325967116683942.

6. Haislup BD, Kraeutler MJ, Baweja R, McCarty EC, Mulcahey MK. Orthopaedic sports medicine fellowship interviews: Structure and organization of the interview day. Orthop J Sports Med 2017;5: 2325967117741276.

7. Yin BB, Ghandi J, Mohr K, ElAttrache NS. Impact of fellowship training on clinical practice of orthopaedic sports medicine. J Bone Joint Surg Am 2015;97:e27.

8. DeFroda SF, Shah KN, Safdar O, Mulcahey MK. Trends in research productivity of residents applying for orthopedic sports medicine fellowship. Phys Sportsmed 2018;46:61-65.

9. Cvetanovich GL, Saltzman BM, Chalmers PH, Frank RM, Cole BJ. Back BR. Research productivity of sports medicine fellowship faculty. Orthop J Sports Med 2016;4: 2325967116679393.

10. Orthogate. Topic: Sports medicine fellowship reviews-new. 2017, https://www.orthogate.org/forums/ orthopedic-surgery-fellowship-forum/5892-sports-medicine-fellowship-reviews-new. Accessed December 28, 2019.

11. Judge PD, Alvi SA, Tawfik KO. Priorities in the neurotology fellowship match: A survey study of program directors and fellows. Ann Otol Rhinol Laryngol 2018;127: 625-630.

12. Yung KC, Courey MS. Factors important in laryngology fellow and laryngology fellowship selection. Laryngoscope 2015;125:2543-2546.

13. Chun R, Preciado D, Brown DJ, et al. Choosing a fellow or fellowship: A survey of pediatric otolaryngologists. JAMA Otolaryngol Head Neck Surg 2014;140:102-105.

14. Klein G, Hussain N, Sprague S, Mehlman CT, Dogbey G, Bhandari M. Characteristics of highly successful orthopedic surgeons: A survey of orthopedic chairs and editors. Can J Surg 2013;56:192-198. 